UDC 514

\title{
A SPECTRAL SEQUENCE IN SURGERY
}

\author{
I. KHEMBLTON [I. HAMBLETON] AND A. F. KHARSHILADZE
}

\begin{abstract}
A spectral sequence whose differentials are connected with iterated BrowderLivesay invariants is constructed. Some examples are considered. Bibliography: 16 titles.
\end{abstract}

It has been known for some time that iterated Browder-Livesay invariants resemble the differentials in a spectral sequence (see [2], [3], [4], [7], [8], [14]). S. Cappell and J. Shaneson in their paper [3] asked: what is this "spectral sequence"?

In this paper we provide an answer by identifying the iterated Browder-Livesay invariants with the differentials of the homotopy spectral sequence of a suitable filtration on $L$-theory and begin to investigate its properties.

Recall that if $G$ is a group with given orientation character $w: G \rightarrow\{ \pm 1\}$, and $\pi \subset G$ is a subgroup of index 2 , then the Browder-Livesay groups $L N_{n}(\pi \rightarrow G)$ are defined (by W. Browder and G. Livesay in [1] for $\pi=1$, and in general by C. T. C. Wall in [11]). These groups appear as the obstruction groups for one-sided submanifold splitting problems.

Let $Y$ be a manifold with $\pi_{1}(Y)=G$ and $w_{1}(Y)=w$. Consider a mapping $\varphi: Y \rightarrow R P^{N}, N>2 \operatorname{dim} Y$, which induces an epimorphism of fundamental groups and is transversal to the projective subspace $R P^{N-1} \subseteq R P^{N}$. Let

$$
\operatorname{ker}\left\{\varphi_{*}: \pi_{1}(Y) \rightarrow \mathbf{Z} / 2\right\}=\pi
$$

and denote by $X=\varphi^{-1}\left(R P^{N-1}\right)$ the induced one-sided codimension 1 submanifold. Assume that $n=\operatorname{dim} X \geq 4$ and that the inclusion $X \subset Y$ induces an isomorphism of fundamental groups, as one can arrange by deforming the map $\varphi$. If $f: N \rightarrow Y$ is a simple homotopy equivalence of closed manifolds, an obstruction in the group $L N_{n}(\pi \rightarrow G)$ is defined, which is zero if and only if the map $f$ is splittable along $X \subset Y$. If $N, Y$ have boundaries assume that $f$ is split along the boundary, and then the obstruction is also defined.

There are two natural maps, which provide a connection between the BrowderLivesay groups and the Wall groups $L_{n}(G)$ :

$$
c: L N_{n}(\pi \rightarrow G) \rightarrow L_{n}\left(G^{-}\right)
$$

and

$$
\partial: L_{n+2}(G) \rightarrow L N_{n}(\pi \rightarrow G) .
$$

Here $G^{-}$is the same as $G$ as a group, but the orientation character of $G^{-}$is defined by $w \phi$, where $\phi: G \rightarrow \mathbf{Z} / 2$ is the epimorphism with kernel $\pi$.

(i) If $x \in L N_{n}(\pi \rightarrow G)$ is the splitting obstruction for some homotopy equivalence $f: N \rightarrow Y$ along $X$, then $c(x) \in L_{n}\left(G^{-}\right)$is the surgery obstruction for $f^{-1}(X) \rightarrow$ $X$.

(ii) If $y \in L_{n+2}(G)$ is the surgery obstruction for normal map $F:(W, \partial W) \rightarrow$

1991 Mathematics Subject Classification. Primary 55T99, 57R67; Secondary 18 F25.

The first author's research was partially supported by NSERC grant A4000. 
$(V, \partial V)$ of manifolds with boundaries, and $\partial V=Y$, then $\partial(y)$ is the splitting obstruction for the homotopy equivalence $F \mid \partial W: \partial W \rightarrow Y$.

All natural maps between different Wall and Browder-Livesay groups can be located in the following braid $(i: \pi \rightarrow G$ is the inclusion):

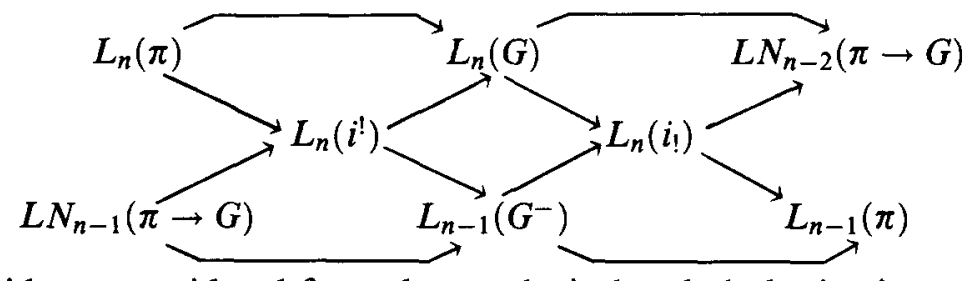

This braid was considered from the topological and algebraic viewpoints in [11], [4], [6], [9], [10], [12]; it is the main tool for the construction of iterated BrowderLivesay invariants.

Recall that all groups of this braid can be realized as the homotopy groups of Quinn-Ranicki spectra $\mathscr{L}(G), \mathscr{L} \mathscr{N}(\pi \rightarrow G)$, etc; see [13]. Note that $\mathscr{L}_{i}(G)=$ $\Omega \mathscr{L}_{i-1}(G)$, so the labeling of the spaces (which are rather not spaces but semisimplicial sets) of these spectra corresponds to the dimension of manifolds. The whole braid (1) can be realized on the level of Quinn-Ranicki spectra, but in fact it is sufficient to consider only the middle square of this braid (for simplicity take $n=0$ ) :

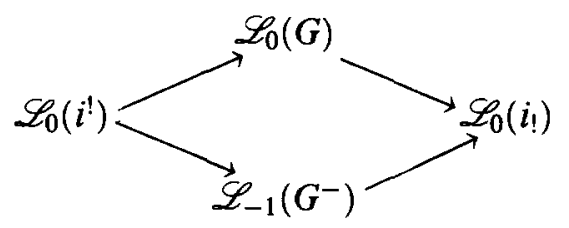

This square is homotopy pull-back (or push-out; this is the same for spectra), and the braid can be obtained just from this square if one takes into account that homotopy fibers (or cofibers) of parallel maps in the square coincide. There exists also a similar square for $G^{-}$instead of $G$. Let us introduce the notation $i_{+}: \pi \subset G$ and $i_{-}: \pi \subset G^{-}$.

Now we shall follow the well-known procedure for construction of the iterated Browder-Livesay invariants, but we shall do it on the level of spaces. Denote

$$
\begin{gathered}
X_{00}=\mathscr{L}_{0}(G), \\
X_{1,1}=\mathscr{L}_{-1}\left(G^{-}\right), X_{2,2}=\mathscr{L}_{-2}(G)=\sum^{2} X_{00}=\cdots, X_{k, k}=\mathscr{L}_{-k}\left(G^{(-)^{k}}\right)=\cdots, \\
X_{1,0}=\mathscr{L}_{0}\left(i_{-}^{!}\right), X_{2,1}=\mathscr{L}_{-1}\left(i_{+}^{!}\right), \ldots, X_{k, k-1}=\mathscr{L}_{-k+1}\left(i_{(-1)^{k}}^{!}\right), \ldots, \\
X_{-1,0}=\mathscr{L}_{0}\left(i_{+!}\right), X_{0,1}=\mathscr{L}_{-1}\left(i_{-!}\right), \ldots, X_{k, k+1}=\mathscr{L}_{-k-1}\left(i_{(-)^{k+l_{1}}}\right), \ldots
\end{gathered}
$$

We have maps

$$
X_{1,0} \rightarrow X_{1,1} \leftarrow X_{2,1}
$$

Denote the homotopy pull-back of these maps by $X_{2,0}$; we obtain the homotopy pull-back square. Also denote by $X_{3,1}$ the homotopy pull-back of the maps

$$
X_{2,1} \rightarrow X_{2,2} \leftarrow X_{3,2}
$$

Proceeding in the same way one obtains the following diagram which consists of 
pull-back squares, and can be extended to the left, up and down:

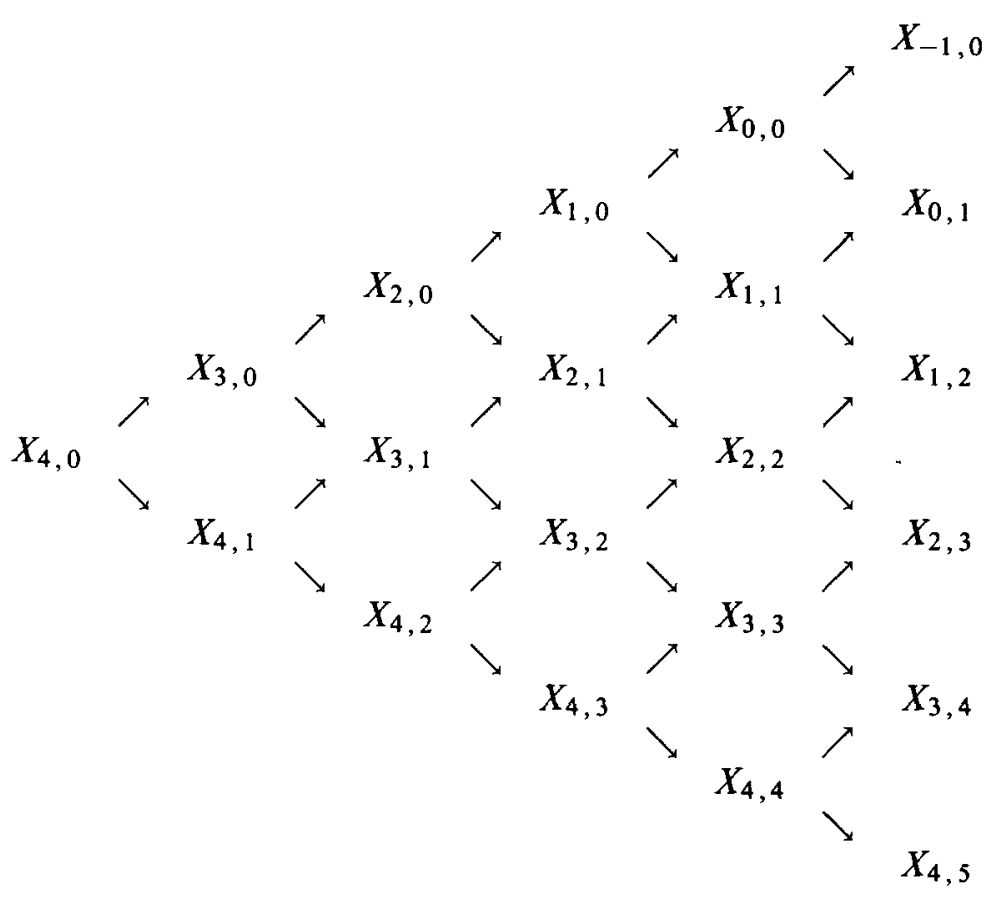

In this diagram the Quinn-Ranicki spectra $\mathscr{L}_{m}\left(G^{ \pm}\right)$are placed in the second column from the right side, and are marked by two equal subscripts. The diagram can also be extended to the right side by the homotopy push-out construction.

In this diagram the relative homotopy groups

$$
\pi_{n}\left(X_{k, i}, X_{k+j, i}\right) \text { and } \pi_{n}\left(X_{i, k}, X_{i, k-j}\right)
$$

for $j>0$ do not depend on $i$ because of excision. And because of this the natural mapping of the filtration

$$
X_{\infty, i} \rightarrow \cdots \rightarrow X_{n, i} \rightarrow X_{n-1, i} \rightarrow \cdots \rightarrow X_{-\infty, i}
$$

into the filtration $X_{\infty, i+1} \rightarrow \cdots \rightarrow X_{n, i+1} \rightarrow X_{n-1, i+1} \rightarrow \cdots \rightarrow X_{-\infty, i+1}$ induces an isomorphism of the homotopy spectral sequences. So the whole spectral sequence of the filtration which is infinite in both directions does not depend on $i$. There is also another infinite filtration with respect to a second subscript, and its homotopy spectral sequence does not depend on the first subscript either. then

Let $E_{1}^{p, q}=\pi_{q-p}\left(X_{p, 0}, X_{p+1,0}\right)=\pi_{q-p}\left(X_{p, i}, X_{p+1, i}\right)$ for any $i$. Take $i=p$;

$$
\begin{aligned}
E_{1}^{p, q} & =\pi_{q-p}\left(X_{p, p}, X_{p+1, p}\right)=\pi_{q-p}\left(\mathscr{L} \mathcal{N}_{-p-2}\left(\pi \rightarrow G^{(-)^{p}}\right)\right) \\
& =L N_{q-2 p-2}\left(\pi \rightarrow G^{(-)^{p}}\right)=L N_{q+2}(\pi \rightarrow G)
\end{aligned}
$$

because $L N_{k}\left(\pi \rightarrow G^{-}\right)=L N_{k+2}(\pi \rightarrow G)$.

Now let us look for the differential $d_{1}: E_{1}^{p, q} \rightarrow E_{1}^{p+1, q}$ given by

$$
d_{1}: L N_{q-2 p-2}\left(\pi \rightarrow G^{(-)^{p}}\right) \rightarrow L N_{q-2 p}\left(\pi \rightarrow G^{(-)^{p+1}}\right)=L N_{q-2 p-2}\left(\pi \rightarrow G^{(-)^{p}}\right) .
$$

From (3) one can see that $d_{1}$ is the same as the composition

$$
L N_{q-2 p-2}\left(\pi \rightarrow G^{(-)^{p}}\right) \stackrel{c}{\longrightarrow} L N_{q-2 p-2}\left(\pi \rightarrow G^{(-)^{p+1}}\right) \stackrel{\partial}{\longrightarrow} L N_{q-2 p}\left(\pi \rightarrow G^{(-)^{p}}\right)
$$


considered in [4]. Recall that algebraically the group $L N_{k}(\pi \rightarrow G)$ can be identified as a Wall group $L_{k}(\mathbf{Z} \pi, \alpha, u)$ over the ring with antistructure $(Z \pi, \alpha, u)$, (see [11], $12 \mathrm{C})$. Here $\alpha: \mathbf{Z} \pi \rightarrow \mathbf{Z} \pi$ is the involution defined by $\alpha(x)=t^{-1} \bar{x} t, u=-w(t) t^{-2}$, where $t \in G-\pi$ is any fixed element. Define $\Phi: L_{k}(\mathbf{Z} \pi, \alpha, u) \rightarrow L_{k}(\mathbf{Z} \pi, \alpha, u)$ by a map on the quadratic category which sends any quadratic form $(M, f)$ over $\left(\mathbf{Z}_{\pi}, \alpha, u\right)$ to the form $(M \otimes t, \bar{f})$, where $M \otimes t$ is the same abelian group as $M$, $(m \otimes t) x=m\left(t x t^{-1}\right) \otimes t$, and

$$
\bar{f}(m \otimes t, n \otimes t)=w(t) t^{-1} f(m, n) t .
$$

In fact the mapping $\Phi$ is an involution on $L_{k}(Z \pi, \alpha, u)$, since $\Phi^{2}$ is induced by an inner automorphism of $\mathbf{Z} \pi$. The composition

$$
\partial c: L N_{k}\left(\pi \rightarrow G^{ \pm}\right) \rightarrow L N_{k-2}\left(\pi, G^{\mp}\right)=L N_{k}\left(\pi \rightarrow G^{ \pm}\right)
$$

is the same as $1 \mp \Phi$ (see [4]). We can see now that

$$
E_{2}^{p, q}=\operatorname{ker}\left(1+(-1)^{p+1} \Phi\right) / \operatorname{im}\left(1+(-1)^{p} \Phi\right)=\hat{H}_{\Phi}^{p}\left(Z / 2: L N_{q}\left(\pi \rightarrow G^{-}\right)\right),
$$

where the last group is the Tate cohomology group of $L N_{q}\left(\pi \rightarrow G^{-}\right)$with respect to $\mathbf{Z} / 2$-action $\Phi$.

Theorem 1. The differentials $d_{k}: E_{k}^{p, q} \rightarrow E_{k}^{p+k, q+k-1}$ coincide with the set of iterated Browder-Livesay invariants.

Proof. Let $x \in L N_{q-2 p-2}\left(\pi \rightarrow G^{(-)^{p}}\right)$ be some representative of the class $[x] \in E_{k}^{p, q}$. Consider the diagram

$$
\begin{array}{rllll}
L N_{q-2 p-2}\left(\pi \rightarrow G^{(-)^{p}}\right) & \stackrel{c}{\rightarrow} & L_{q-2 p-2}\left(G^{(-)^{p+1}}\right) & \stackrel{\partial}{\rightarrow} & L N_{q-2 p-4}\left(\pi \rightarrow G^{(-)^{p+1}}\right) \\
& & & & \\
L_{q-2 p-3}\left(G^{(-)^{p}}\right) & \stackrel{\partial}{\rightarrow} & L N_{q-2 p-5}\left(\pi \rightarrow G^{(-)^{p}}\right) \\
\vdots & & & \\
& L_{q-2 p-2-i}\left(G^{(-)^{p+1+i}}\right) & \stackrel{\partial}{\rightarrow} & L N_{q-2 p-4-i}\left(\pi \rightarrow G^{(-)^{p+1+i}}\right)
\end{array}
$$

If $\partial c x=d_{1} x=0$ then a coset $\Gamma(x) \subset L_{q-2 p-3}\left(G^{(-)^{p}}\right)$ is defined (see [14]). If the coset $\partial(\Gamma(c x)) \subset L N_{q-2 p-5}\left(\pi \rightarrow G^{(-)^{p}}\right)$ contains zero, then the coset

$$
\Gamma^{2}(c x) \subset L_{q-2 p-4}\left(G^{(-)^{p+1}}\right)
$$

is defined. The differential $d_{k}$ is the same as $\partial^{k-1} c(x)$, and this is just the definition of the iterated Browder-Livesay invariants.

From the diagram (3) one can see that $d_{k}$ is the same as $\partial \Gamma^{k-1}(c x)$, the last being the definition of the iterated Browder-Livesay invariants. In a similar way denote

$$
F_{1}^{p, q}=\pi_{p+q}\left(X_{0, p}, X_{0, p-1}\right)=\pi_{p+q}\left(X_{p, p}, X_{p, p-1}\right)=\pi_{p+q}\left(\mathscr{L}_{-p}(\pi)\right)=L_{q}(\pi),
$$

where $d_{1}^{\prime}: F_{1}^{p, q}=L_{q}(\pi) \rightarrow F^{p-1, q}=L_{q}(\pi)$ is the composition

$$
L_{q}(\pi) \stackrel{i_{1}}{\rightarrow} L_{q}\left(G^{(-)^{p+1}}\right) \stackrel{! !}{\rightarrow} L_{q}(\pi),
$$

which is equal to $1+(-1)^{p+1} w(t)$. It follows that

$$
F_{2}^{p, q}=H_{w(t)}^{p}\left(\mathbf{Z} / 2 ; L_{q}(\pi)\right) .
$$

The differentials $d_{k}^{\prime}: F_{k}^{p, q} \rightarrow F_{k}^{p-k, q+k-1}$ also have a geometrical interpretation using diagram (1). 
Lemma 2. If the groups $E_{1}^{p, q}$ and $F_{1}^{p, q}$ are finitely generated, then the spectral sequences converge to $\pi_{*}\left(X_{-\infty, 0}, X_{\infty, 0}\right)$ and $\pi_{*}\left(X_{0, \infty}, X_{0,-\infty}\right)$ respectively.

Proof. The proof is based on a similar result of Bousfield and Kan (see [16], p. 263). In [16] the required convergence is proved for a filtration

$$
Y_{\infty} \rightarrow \cdots \rightarrow Y_{n} \rightarrow Y_{n-1} \rightarrow \cdots \rightarrow Y_{1} \rightarrow Y_{0} \rightarrow *
$$

which is infinite only to the left, and we have here a filtration infinite in both directions. Define

$$
A_{i}^{p}=\operatorname{im}\left\{\pi_{*}\left(X_{p, 0}, X_{\infty, 0}\right) \rightarrow \pi_{*}\left(X_{-\infty, 0}, X_{\infty, 0}\right)\right\}
$$

so that $\cdots \subset A_{i}^{p+1} \subset A_{i}^{p} \subset \cdots$ is a filtration of the group $\pi_{*}\left(X_{-\infty, 0}, X_{\infty, 0}\right)$ infinite in both directions. Now the assertion of the lemma is

(a) $\bigcap_{p=-\infty}^{\infty} A_{i}^{p}=0$,

(b) $\bigcup_{p=-\infty}^{\infty} A_{i}^{p}=\pi_{i}\left(X_{-\infty, 0}, X_{\infty, 0}\right)$,

(c) $A_{i}^{p} / A_{i}^{p-1}=E_{\infty}^{p, p+i}$.

Let us restrict now the filtration from the right, and consider the filtration

$$
X_{\infty, 0} \rightarrow \cdots \rightarrow X_{p, 0} \rightarrow X_{p-1,0} \rightarrow \cdots \rightarrow X_{0,0} \rightarrow X_{-1,0} \rightarrow \cdots \rightarrow X_{M, 0} .
$$

Let $\left(\bar{E}_{k}^{p, q}, \bar{d}_{k}\right)$ be the homotopy spectral sequence, and let us denote

$$
B_{i}^{p}=\operatorname{im}\left\{\pi_{i}\left(X_{p}, 0, X_{\infty, 0}\right) \rightarrow \pi_{i}\left(X_{M, 0}, X_{\infty, 0}\right)\right\} .
$$

Following Bousfield and Kan, we have

(a') $\bigcap_{p=-\infty}^{M} B_{i}^{p}=0$;

(b) $\bigcup_{p=-\infty}^{\infty} B_{i}^{p}=\pi_{i}\left(X_{M, 0}, X_{\infty, 0}\right)$;

(c) $B_{i}^{p} / B_{i}^{p+1}=\bar{E}_{\infty}^{p, p+i}=\bar{E}_{N}^{p, p+1}$, for some $N$

i.e. under the conditions in the lemma we have Mittag-Leffler convergence.

By the telescopic construction of the space $X_{-\infty, 0}$ a standard argument shows that each element of $\pi_{*}\left(X_{-\infty, 0}, X_{\infty, 0}\right)$ is in the image of $\pi_{*}\left(X_{M, 0}, X_{\infty, 0}\right)$ for some $M$. Moreover, if the image of some element of $\left.\pi_{*}\left(X_{M}, 0\right), X_{\infty, 0}\right)$ in $\pi_{*}\left(X_{-\infty, 0}, X_{\infty, 0}\right)$ is trivial, then its image is also trivial in $\pi_{*}\left(X_{M^{\prime}, 0}, X_{\infty}, 0\right)$, for some $M^{\prime}<M$. This implies assertion (b). Now the natural map $B_{i}^{p} \rightarrow A_{i}^{p}$ is onto for $M<p$, and so (a') implies (a).

For all $p>M+N$ and $r>N$ the differentials $\bar{d}_{r}: \bar{E}_{r}^{p, q} \rightarrow \bar{E}_{r}^{p+r, q+r-1}$ are trivial, and so $E_{\infty}^{p, q}=E_{N}^{p, q}=\bar{E}_{N}^{p, q}=\bar{E}_{\infty}^{p, q}$. For (c) it is sufficient to show that for some $M$, which must be negative and of large absolute value,

$$
B_{i}^{p} / B_{i}^{p+1}=A_{i}^{p} / A_{i}^{p+1} \text {. }
$$

The natural map $B_{i}^{p} / B_{i}^{p+1} \rightarrow A_{i}^{p} / A_{i}^{p+1}$ is onto, and if $x \in B_{i}^{p} / B_{i}^{p+1}$ is in the kernel of this map, then (again by the telescope construction) $x$ will become zero if we make $M$ large negative. One can make the value of $M$ needed independent of $x$, because $B_{i}^{p} / B_{i}^{p+1}=\bar{E}_{N}^{p, p+i}$ is finitely generated. This proves assertion (c) and Lemma 2.

Let us again restrict the filtration from above, and consider the part

$$
X_{\infty, 0} \rightarrow \cdots \rightarrow X_{n, 0} \rightarrow X_{n-1,0} \rightarrow \cdots \rightarrow X_{0,0}=L_{0}(G) .
$$

The homotopy spectral sequence $\left({ }^{\prime} E^{p, q}, d_{*}\right)$ of this filtration maps epimorphically ${ }^{\prime} E_{k}^{p, q} \rightarrow E_{k}^{p, q}$ onto the spectral sequence of the filtration which is unrestricted in both directions if $p \geq 0$, and for $p<0$ all groups ' $E_{k}^{p, q}$ are trivial. 
If the Browder-Livesay groups $L N_{*}\left(\pi \rightarrow G^{ \pm}\right)$are finitely generated this spectral sequence converges to $\pi_{*}\left(X_{0,0}, X_{\infty, 0}\right)$. There is a natural mapping

$$
D: L_{n}(G)=\pi_{n}\left(X_{0,0}\right) \rightarrow \pi_{n}\left(X_{0,0}, X_{\infty}, 0\right)
$$

By Theorem 1 this mapping coincides with the whole set of iterated Browder-Livesay invariants.

Proposition 3. An element $x \in L_{n}(G)$ is an element of the first type (in the sense of [14]) with respect to a subgroup $\pi \subset G$ if and only if $D(x) \neq 0$.

Proposition 4. If $E_{\infty}=0$ and the groups $L N_{*}\left(\pi \rightarrow G^{ \pm}\right)$are finitely generated, then the mapping $D$ is surjective.

Proof. It follows that the map $\pi_{*}\left(X_{0,0}, X_{\infty, 0}\right) \rightarrow \pi_{*}\left(X_{-k, 0}, X_{\infty, 0}\right)$ is trivial for sufficiently large $k>0$. Now surjectivity of $D$ follows from the commutative diagram with the exact columns

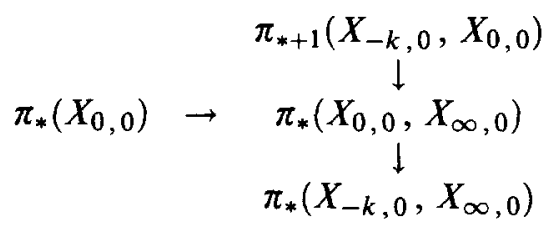

The image of the map $\pi_{*}\left(X_{\infty, 0}\right) \rightarrow \pi_{*}\left(X_{0,0}\right)=L_{*}(G)$ consists of the elements of the second and third type in the sense of [14] with respect to the subgroup $\pi \subset G$.

Proposition 5. An element $x \in \pi_{*}\left(X_{0,0}\right)=L_{*}(G)$ is an element of the third type if and only if

$$
x \in \operatorname{im}\left[\pi_{*}\left(X_{\infty, 0}\right) \rightarrow \pi_{*}\left(X_{0,0}\right)\right] \cap \operatorname{ker}\left[\pi_{*}\left(X_{0,0}\right) \rightarrow \pi_{*}\left(X_{0, \infty}\right)\right],
$$

and it is of the second type if and only if

$$
x \in \operatorname{im}\left[\pi_{*}\left(X_{\infty, 0}\right) \rightarrow \pi_{*}\left(X_{0,0}\right)\right]
$$

but $x \notin \operatorname{ker}\left[\pi_{*}\left(X_{0,0}\right) \rightarrow \pi_{*}\left(X_{0, \infty}\right)\right]$.

Being in the kernel of $\pi_{*}\left(X_{0,0}\right) \rightarrow \pi_{*}\left(X_{0, \infty}\right)$ is the same as being in the kernel of $\pi_{*}\left(X_{0,0}\right) \rightarrow \pi_{*}\left(X_{0, k}\right)$ for sufficiently large $k>0$, and this is the same as being the image of some differential of homotopy spectral sequence of the "restricted from below" filtration

$$
\{*\} \subset X_{0,0} \rightarrow X_{0,1} \rightarrow X_{0,2} \rightarrow \cdots \rightarrow X_{0, k} \rightarrow \cdots .
$$

For this spectral sequence, ' $F_{1}^{p, q}=F_{1}^{p, q}$ if $p>0,{ }^{\prime} F_{1}^{0, q}=L_{q}(G)$, and ${ }^{\prime} F_{1}^{p, q}=0$ if $p<0$.

Now we give some examples. The groups $E_{1}^{p, q}$ do not depend on $p$ and are 4-periodic in $q$. But for $k>1$ the groups $E_{k}^{p, q}$ are 4-periodic in $q$ and 2-periodic in $p$. It is convenient to write these groups $E_{1}^{p, q}$ in tabular form

$$
E_{1}^{p, q}=\begin{array}{|c|c|c|c|}
\hline L N_{0}(\pi \rightarrow G) & L N_{3}\left(\pi \rightarrow G^{-}\right) & L N_{2}(\pi \rightarrow G) & L N_{2}\left(\pi \rightarrow G^{-}\right) \\
\hline L N_{2}\left(\pi \rightarrow G^{-}\right) & L N_{1}(\pi \rightarrow G) & L N_{0}\left(\pi \rightarrow G^{-}\right) & L N_{3}(\pi \rightarrow G) \\
\hline
\end{array}
$$

Here the groups $E_{1}^{p, q}$ are not placed each in the cell with $(p, q)$ coordinates, although each stands in the right place with respect to the $q$-coordinate. In this table the differential $d_{1}$ acts vertically up or down, so the domain and range are related by a change in position of $(0, \pm 1)$. Similarly, $d_{2}$ changes position by $(+1, \pm 1)$, $d_{3}$ by $(+2, \pm 1)$, and so on (mod 4 in the horizontal direction). 
We begin with finite abelian 2-groups. The Browder-Livesay groups $L N_{n}(\pi \rightarrow G)$ for this case (with prime decoration) are given in [12], as well as a lot of information about the natural maps between Wall and Browder-Livesay groups. Denote as usual by $r$ the 2-rank of $G$, and by $s$ the number of $Z / 2$ direct summands in $G$. There are three cases:

1) $\left.w\right|_{2} \pi$ is nontrivial $\left({ }_{2} \pi=\operatorname{ker}[\pi \stackrel{\times 2}{\rightarrow} \pi]\right)$ :

$$
\begin{aligned}
& L N_{2 n+1}(\pi \rightarrow G)=(\mathbf{Z} / 2)^{2^{r-1}-s-1}, \\
& L N_{2 n}(\pi \rightarrow G)=(\mathbf{Z} / 2)^{2^{r-1}-s} .
\end{aligned}
$$

2) $\pi \subseteq \operatorname{ker} w:$

$$
\begin{aligned}
& L N_{2 n+1}(\pi \rightarrow G)=(\mathbf{Z} / 2)^{2^{r}-1-r-\left(\begin{array}{l}
s \\
2
\end{array}\right),} \\
& L N_{2 n}(\pi \rightarrow G)=\left\{R(G) / i_{*} R(\pi)\right\} /(\approx) \oplus(\mathbf{Z} / 2)^{2^{r-1}-r-\left(\begin{array}{l}
s \\
2
\end{array}\right),}
\end{aligned}
$$

where $R(G)$ is the group of complex representation, $i_{*}: R(\pi) \rightarrow R(G)$ is the induction map, and in the above formula orbits under complex conjugation are considered.

3) $\left.w\right|_{2} \pi$ trivial, but $w \mid \pi$ nontrivial:

$$
\begin{aligned}
& L N_{2 n+1}(\pi \rightarrow G)=(\mathrm{Z} / 2)^{2^{r}+2^{r-2}-r-1-\left(\begin{array}{l}
s \\
2
\end{array}\right),} \\
& L N_{2 n}(\pi \rightarrow G)=(\mathbf{Z} / 2)^{2^{r-1}-r-\left(\begin{array}{l}
s \\
2
\end{array}\right) .}
\end{aligned}
$$

With these formulas, for any abelian group $G$ and any index two subgroup $\pi$ one can write down the $E_{1}$ term. In case of abelian groups $\Phi= \pm 1$ and $d_{1}=1 \pm 1$, and so the torsion part goes to the $E_{2}$-term unchanged. In fact in cases 1) and 3) when the $L N$-groups do not contain a free part, $E_{1}=E_{\infty}$ (see the diagrams at the end of [12]).

Consider the only remaining case, 2 ). For short let us denote

$$
\begin{aligned}
& A=(\mathrm{Z} / 2)^{2^{r}-1-r-\left(\begin{array}{l}
s \\
2
\end{array}\right)}=L N_{2 n+1}(\pi \rightarrow G), \\
& F=\left\{\text { free part of } L N_{2 n}(\pi \rightarrow G)\right\}=\left\{R(G) / i_{*} R(\pi)\right\} /(\approx) \text {, } \\
& B=\left\{\text { torsion of } L N_{2 n}(\pi \rightarrow G)\right\}=(Z / 2)^{2^{r-1}-r-\left(\begin{array}{l}
s \\
2
\end{array}\right) .}
\end{aligned}
$$

Note that the orientation character is trivial for $G$, and nontrivial with kernel $\pi$ for $G^{-}$.

$$
\begin{aligned}
& E_{1}^{p, q}=\begin{array}{|c|c|c|c|}
\hline F \oplus B & A & F \oplus B & A \\
\hline F \oplus B & A & F \oplus B & A \\
\hline
\end{array} \\
& E_{1}^{p, q}=\begin{array}{|c|c|c|c|}
\hline F / 2 F \oplus B & A & F / 2 F \oplus B & A \\
\hline B & A & B & A \\
\hline
\end{array}
\end{aligned}
$$

and $E_{2}=E_{\infty}$ since all differentials $d_{r}$ with $r>1$ are trivial. This is not so easy to show in the case of

$$
d_{2}: L N_{3}(\pi \rightarrow G) \rightarrow L N_{0}(\pi \rightarrow G) / \mathrm{im} d_{1},
$$

but this was done recently by $\mathrm{Yu}$. V. Muranov. For abelian 2-groups the conclusion is that all differentials except $d_{1}$ are trivial.

However, in the nonabelian case nontrivial $d_{2}$ differentials do occur. For example let $D$ be a dihedral group of order $2 d, C$ a cyclic index 2 subgroup of $D$, and assume that $d$ is odd. 
Let us begin with $L^{K}$-theory using the decoration and 2-adic coefficients. According to [15], §4:

$$
\begin{aligned}
& L_{n}^{K}\left(\widehat{\mathbf{Z}}_{2}(C)\right)(d)=0 \\
& L N_{n}^{K}\left(\widehat{\mathbf{Z}}_{2}(C \rightarrow D)\right)(d)=(\mathbf{Z} / 2)^{\tilde{g}_{2}} \\
& L_{n}^{K}\left(\widehat{\mathbf{Z}}_{2}\left(D^{ \pm}\right)\right)(d)=(\mathbf{Z} / 2)^{g_{2}} .
\end{aligned}
$$

Here the letter $d$ in parentheses on the right of each group means the "top components" in the sense of [5], and

$$
g_{2}=\left[(\mathbf{Z} / d)^{\times}:\langle 2,-1\rangle\right], \quad \tilde{g}_{2}=\left[(\mathbf{Z} / d)^{\times}:\langle 2\rangle\right] .
$$

We have the isomorphism $\Gamma$ of homology groups of two chain complexes

$$
\begin{gathered}
L_{0}(\pi) \rightarrow L_{0}(G) \rightarrow L N_{2}(\pi \rightarrow G) \\
\downarrow \\
L \Gamma \\
L N_{3}(\pi \rightarrow G) \rightarrow L_{3}\left(G^{-}\right) \rightarrow L N_{3}(\pi \rightarrow G)
\end{gathered}
$$

In our case both the maps $L N_{3}^{K}\left(\widehat{\mathbf{Z}}_{2}\left(C \rightarrow D^{-}\right)\right)(d) \rightarrow L_{3}^{K}\left(\widehat{\mathbf{Z}}_{2}(D)\right)(d)$ and the composite $\partial \circ \Gamma$ from $L_{0}^{K}\left(\widehat{\mathbf{Z}}_{2}\left(D^{-}\right)\right)(d) \rightarrow L N_{3}^{K}\left(\widehat{\mathbf{Z}}_{2}(C \rightarrow D)\right)(d)$ are trivial. This follows immediately from $(2.17)$ of [5]. The groups $L_{0}^{K}\left(\widehat{\mathbf{Z}}_{2}(C)\right)(d)$ and $L_{3}^{K}\left(\widehat{\mathbf{Z}}_{2}(C)\right)(d)$ are trivial, and so the homology isomorphism becomes an isomorphism of the groups $L_{0}^{K}\left(\widehat{\mathbf{Z}}_{2}\left(D^{-}\right)\right)(d)$ and $L_{3}^{K}\left(\widehat{\mathbf{Z}}_{2}\left(D^{-}\right)\right)(d)$ themselves: both groups are isomorphic to $(Z / 2)^{g_{2}}$. Now from 2.10 of [5] we have that

$$
L N_{2 k}^{K}\left(\widehat{\mathbf{Z}}_{2}(C \rightarrow D)\right)(d) \rightarrow L_{2 k}^{K}\left(\widehat{\mathbf{Z}}_{2}\left(D^{-}\right)\right)(d)
$$

is a split surjection and can be identified with the projection

$$
(\mathbf{Z} / 2)^{\left.[\mathbf{Z} / d)^{\times}:\langle 2\rangle\right]} \rightarrow(\mathbf{Z} / 2)^{\left[(\mathbf{Z} / d)^{\times}:\langle 2,-1\rangle\right]} .
$$

Also from 2.17 of [5] we have that

$$
L_{2 k+1}^{K}\left(\widehat{\mathbf{Z}}_{2}(D)\right)(d) \rightarrow L N_{2 k-1}^{K}\left(\widehat{\mathbf{Z}}_{2}(C \rightarrow D)\right)(d)
$$

is a split injection and can be identified with

$$
\operatorname{Hom}\left((\mathbf{Z} / 2)^{\left[(\mathbf{Z} / d)^{\times}:\langle 2,-1)\right]}, \mathbf{Z} / 2\right) \rightarrow \operatorname{Hom}\left((\mathbf{Z} / 2)^{\left[(\mathbf{Z} / d)^{\times}:(2)\right]}, \mathbf{Z} / 2\right)
$$

Now consider the diagram

$$
\begin{gathered}
L N_{2 k}\left(\widehat{\mathbf{Z}}_{2}(C \rightarrow D)\right)(d) \rightarrow L_{2 k}^{K}\left(\widehat{\mathbf{Z}}_{2}\left(D^{-}\right)\right)(d) \stackrel{0}{\rightarrow} L N_{2 k-2}\left(\widehat{\mathbf{Z}}_{2}\left(C \rightarrow D^{-}\right)\right)(d) \\
\cong \downarrow \Gamma \\
\begin{aligned}
L N_{2 k-1}\left(\widehat{\mathbf{Z}}_{2}\left(C \rightarrow D^{-}\right)\right)(d) \stackrel{0}{\rightarrow} L_{2 k-1}^{K}\left(\widehat{\mathbf{Z}}_{2}\left(D^{-}\right)\right)(d) \rightarrow L N_{1}\left(\widehat{\mathbf{Z}}_{2}(C \rightarrow D)\right)(d) \\
\cong \downarrow \Gamma
\end{aligned}
\end{gathered}
$$

From this diagram one can see that in this case $d_{1}=0$ and

$$
d_{2}: L N_{2 k}^{K}\left(\widehat{\mathbf{Z}}_{2}(C \rightarrow D)\right)(d) \rightarrow L N_{2 k-1}^{K}\left(\widehat{\mathbf{Z}}_{2}(C \rightarrow D)\right)(d)
$$

is an isomorphism whenever $g_{2}=\tilde{g}_{2}$.

We have here examples not only of nontrivial $d_{2}$-differentials but also cases where $E_{\infty}=E_{3}=0$ (see Proposition 4). One can conclude also from [15], 4.3 and 4.5.2, that the map

$$
L N_{0}^{\prime}(C \rightarrow D) \rightarrow L N_{0}^{K}\left(\widehat{\mathbf{Z}}_{2}(C \rightarrow D)\right)(d)
$$

is surjective, and so in the $L^{\prime}$-groups with integral coefficients we also have nontrivial $d_{2}$-differentials. 
Problem. Given $k$, do there exist groups $G$ and $\pi$ with a nontrivial differential $d_{k}$ ?

\section{BIBLIOGRAPHY}

1. W. Browder and G. R. Livesay, Fixed point free involutions on homotopy spheres, Bull. Amer. Math. Soc. 73 (1967), 242-245.

2. S. E. Cappell and J. L. Shaneson, Pseudofree actions. I, Algebraic Topology, Aarkus, 1978, Lecture Notes in Math., vol. 763, Springer-Verlag, Berlin, 1979, pp. 635-642.

3. _ A counterexample on the oozing problem for closed manifolds, Algebraic Topology, Aarkus, 1978, Lecture Notes in Math., vol. 763, Springer-Verlag, Berlin, 1979, pp. 627-639.

4. I. Hambleton, Projective surgery obstructions on closed manifolds, Algebraic K-theory. Part II (Oberwolfach, 1980), Lecture Notes in Math., vol. 967, Springer-Verlag, Berlin, 1982, pp. 101131.

5. I. Hambleton and I. Madsen, Discriminants of forms with Arf invariant one, J. Reine Angew. Math. 395 (1989), 142-166.

6. I. Hambleton, L. Taylor, and B. Williams, An introduction to maps between surgery obstruction groups, Algebraic Topology (Aarkus, 1982), Lecture Notes in Math., vol. 1051, Springer-Verlag, Berlin, 1984, pp. 49-127.

7. A. F. Kharshiladze, Smooth piecewise-linear structures on products of projective spaces, Izv. Akad. Nauk SSSR Ser. Math. 47 (1983), 366-383; English transl. in Math. USSR-Izv. 22 (1984), 339-355.

8. __ Splittings along systems of submanifolds, Mat. Sb. 125(167) (1984), 280-286; English transl. in Math. USSR-Sb. 53 (1986), 283-289.

9. —_ Hermitian $K$-theory and quadratic extensions of rings, Trudy Moskov. Mat. Obshch. 41 (1980), 3-36; English transl. in Trans. Moscow Math. Soc. 1980, no. 1(41), 1-37.

10. A. Ranicki, The L-theory of twisted quadratic extensions, Canad. J. Math. 39 (1987), no. 2, 345364.

11. C. T. C. Wall, Surgery on compact manifolds, Academic Press, New York, 1970.

12. Yu. V. Muranov and A. F. Kharshiladze, Browder-Livesay groups for abelian 2-groups, Mat. Sb. 181 (1990), 1061-1098; English transl. in Math. USSR-Sb. 70 (1991), 449-540.

13. A. Ranicki, The total surgery obstruction, Algebraic Topology, Aarkus, 1978, Lecture Notes in Math., vol. 763, Springer-Verlag, Berlin, 1979, pp. 275-316.

14. A. Kharshiladze, Surgery on manifolds with finite fundamental groups, Uspekhi Mat. Nauk $\mathbf{4 2}$ (1987), no. 4 (256), 55-85; English transl. in Russian Math. Surveys 42 (1987), no. 4, 65-103.

15. C. T. C. Wall, On the classification of Hermitian forms. VI: Group rings, Ann. of Math. (2) 103 (1976), 1-80.

16. A. K. Bousfield and D. M. Kan, Homotopy limits, completions and localizations, Lecture Notes in Math., vol. 304, Springer-Verlag, Berlin, 1972.

Department of Mathematics and Statistics, McMaster University, hamilton, Ontario, CANADA

Institute of Terrestrial Magnetism, Ionosphere and Radio Wave Propagation, Russian ACAdemy of ScIences, TroitsK, Moscow Region, Russia

Received 1/MAR/91

Translated by the first author 